\title{
Experimentation and Structural Modeling of Stimulus-Evoked Electromyography in Muscles During Electrically-Elicited Fatigue Process
}

\author{
Shao-Hsia Chang ${ }^{1}$ and Nan-Ying $\mathrm{Yu}^{2}$ \\ ${ }^{1}$ Department of Occupational Therapy, \\ ${ }^{2}$ Department of Physical Therapy, \\ I-Shou University, Kaohsiung City, \\ Taiwan
}

\section{Introduction}

Functional electrical stimulation (FES) has been adopted to induce muscle contraction to provide functionally useful movements in spinal cord injured (SCI) individuals. It was described as electrical stimulation of muscle deprived of nervous control with a view of providing muscular contraction and providing a functionally useful movement. The term FES is applied to systems which attempt to restore lost or impaired neuromuscular function, such as standing and walking in cases of paraplegia. By the application of electrical pulses to neural pathways or, but less often, directly to muscles. It is also sometimes known as Functional Neuromuscular Stimulation or FNS. Figure 1 shows the demonstration of general applications of FES for the restoration of limb functions. Among all the limiting factors in the application of FES, muscle fatigue that can directly discontinue the application is considered the most important [1]. Many researchers have studied electrically elicited muscle fatigue of paralyzed limb muscles from the biomechanical, metabolic and electrophysiological aspects [2, 3, 4]. During electrical stimulation, the decline of torque values during isometric contraction is usually observed which exhibited a sigmoid-like curve consisting of a main slope and two plateaus [5, 6, 7]. Researchers reported that the first plateau represents the status before fatigue and the major slope indicates the exhausted expenditure of easily-fatigued (type II) MUs. Following the quick fatigue process, the final plateau represents the activation of the residual fatigue-resistant (type I) MUs [8].

From the electrophysiological aspect, the evoked EMG or compound muscle action potential (CMAP) of the stimulated muscle has been studied during the fatiguing process. The close correlation between muscle force and stimulus-evoked EMG peak to peak (PTP) amplitude indicates that the stimulus EMG amplitude could directly provide information for monitoring FES-induced muscle force and as control signals for compensating the decrease of muscle force due to fatigue $[9,10]$. In addition to PTP, the other characteristics of CMAP, such as temporal features including latency, rise time to peak (RTP), and PTP duration (PTPd) as well as frequency characteristics have also been used to quantify the muscle fatigue process $[11,12]$. Both temporal and frequency features were presumably related to 


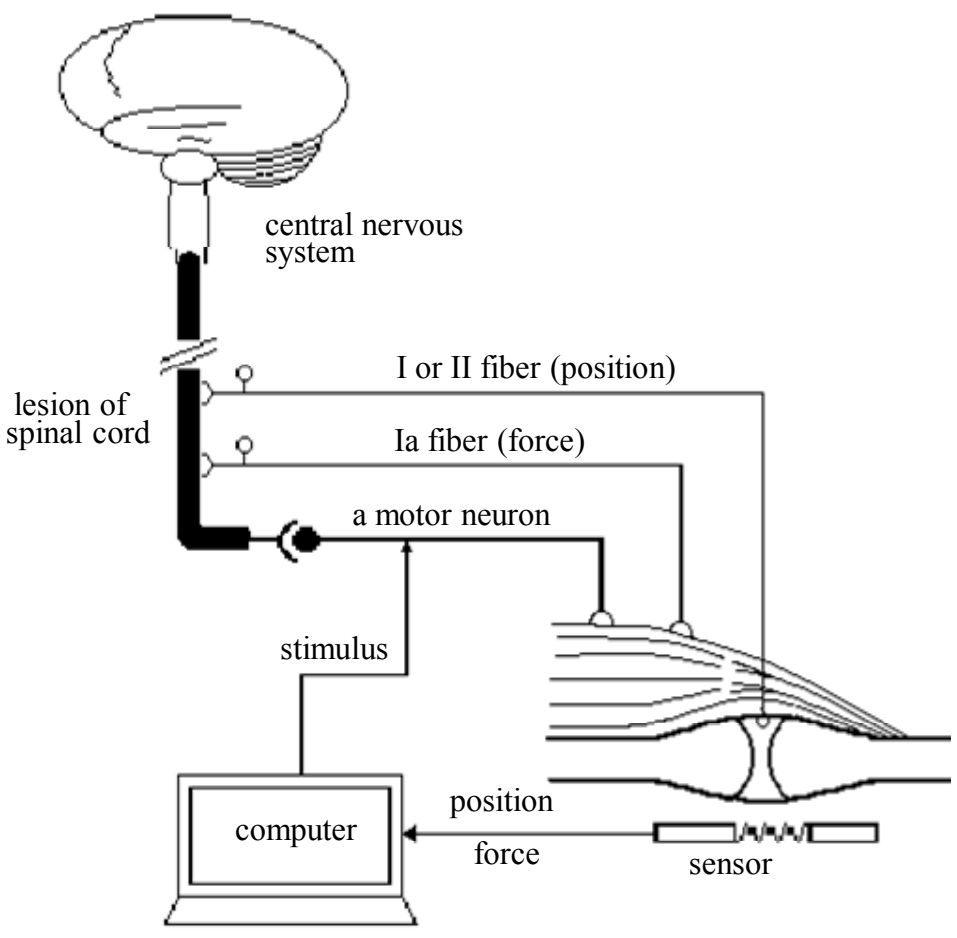

Fig. 1. Illustration of functional electrical stimulation. Arrowed lines show the interface of electrical stimulation and neuromuscular system.

the propagation velocity of motor unit action potential (MUAP) in muscle fibers. Most of the results showed decrease in muscle fiber conduction velocity and reduction in mean/median frequency during the muscle fatigue process [13].

Several studies confirmed that the lengths of stimulated muscle could affect the spectral, temporal, and amplitude features of evoked EMG [13, 14, 15]. Our previous study indicated that the amplitudes of the evoked EMG of the quadriceps muscle in electrically elicited muscle contractions were significantly different when the knee angle was varied [16]. Other studies also indicated that surface EMG features might vary due to the change in relative location between the electrodes and the muscle fibers during stimulation in different muscle lengths which should be taken into consideration for the measurement of evoked EMG [17]. These studies revealed the necessity of electrophysiological studies to illuminate the fatigue characteristics of muscles stimulated in different lengths.

Although studies of the length effects on the fatigue characteristics of stimulated muscles have been proposed, experimental designs to explain some of the intrinsic factors, such as the changes in CMAP amplitude or duration in different muscle fiber types during muscle fatigue process as well as those under different measurement configurations (e.g. varied muscle lengths) are intractable. Thus, a simulation EMG model becomes necessary in describing the meanings of the parameters derived from an evoked EMG, and then verifying their relationships with the fatigue process. For EMG modeling, Fuglevand et al. 
developed a structural MUAP model to investigate the influence of electrode size and spacing [18]. The model of MUAPs has been expanded by Farina et al. in order to consider the effects of electrode configuration, electrode size and the inclination of the fibers with respect to the detection system [14, 19].

The study aim is to combine the experimental design and modeling technique from which the electrophysiological changes of stimulated paralyzed muscle during fatigue process can be interpreted from the amplitude and temporal features of CMAP and its muscle fiber constituents. This study first compared the changes in amplitude and temporal features of CMAP and torque output between the shortened and lengthened paralyzed muscles. Due to the lack of a MUAP model dedicated to SCI paralyzed muscles, we modified the structural MUAP model developed by Fuglevand et al. [18], by incorporating the viable parameters obtained from paralyzed muscles, mainly on the different distributions in MU types and their simulation parameters. Based on the modified structural CMAP model, the stimulus-evoked EMG can be modeled by summing up all the MUAPs together with the parameters representing the characteristics of slow-twitch (type I) and fast-twitch (type II) motor units.

\section{Methods}

\subsection{Experimental protocol}

Six spinal cord injured subjects (5 males and 1 female) with mean \pm SD age of 38.6 $\pm 7.5 \mathrm{yr}$ were recruited for this experiment. The average time post-injury was $6.3 \pm 0.45$ years. The neurological levels of the subjects are between C7 and T11 and with little or no spasticity in the lower limb muscles. All subjects had no musculoskeletal problems in their lower extremities. All subjects signed an informed consent form approved by National Cheng Kung University Hospital, Tainan, Taiwan.

During the muscle fatigue study, stimulation electrodes $(5 \mathrm{~cm} \times 10 \mathrm{~cm})$ were placed to the distal and proximal ends of the quadriceps muscle bellies. The anode was placed $5 \mathrm{~cm}$ above the upper border of the patella. The cathode was placed $20 \mathrm{~cm}$ proximal to the placement of anode. During the experiment, the subject was seated in the chair of an electrodynamometer (Cybex 6000, Lumex, Inc., NY, USA), with his or her hip joint fixed in an $80^{\circ}$ flexion. The muscle group was stimulated by a constant current stimulator with a monophasic waveform at $20 \mathrm{~Hz}$ and pulse duration of $300 \mu \mathrm{sec}$ for 120 seconds. Figure 2 shows the apparatuses for the experiment and data acquisition. Muscle fatigue study was performed at two knee flexion angles at a selected stimulation intensity when the torque output of stimulated muscle reached a stable plateau or when the intensity reached the maximum of $120 \mathrm{~mA}$. The two knee flexion angles were $30^{\circ}$ (short length) and $110^{\circ}$ (long length) with reference to knee full extension at $0^{\circ}$. In this arrangement, the muscles can be stimulated in both comparatively short and long positions. In addition to the stimulus-evoked EMG, the generated torque was also recorded during isometric tests. Adequate rest (at least 24 hours) was required to avoid a possible carryover effect due to a short recovery time after muscle fatigue [1].

To detect the stimulus-evoked EMG, active bipolar surface electrodes with preamplifiers (Motion Control Co., Salt Lake City, Utah, USA) were placed on the RF muscle belly. The recording electrodes were placed inside the stimulation electrodes and mid-way between the anterior superior iliac spine (ASIS) and the upper border of the patella [20]. The interelectrode distance was $20 \mathrm{~mm}$ and the electrode position was marked between sessions and maintained in each subject throughout the experiments. Figure 3 shows the placement 


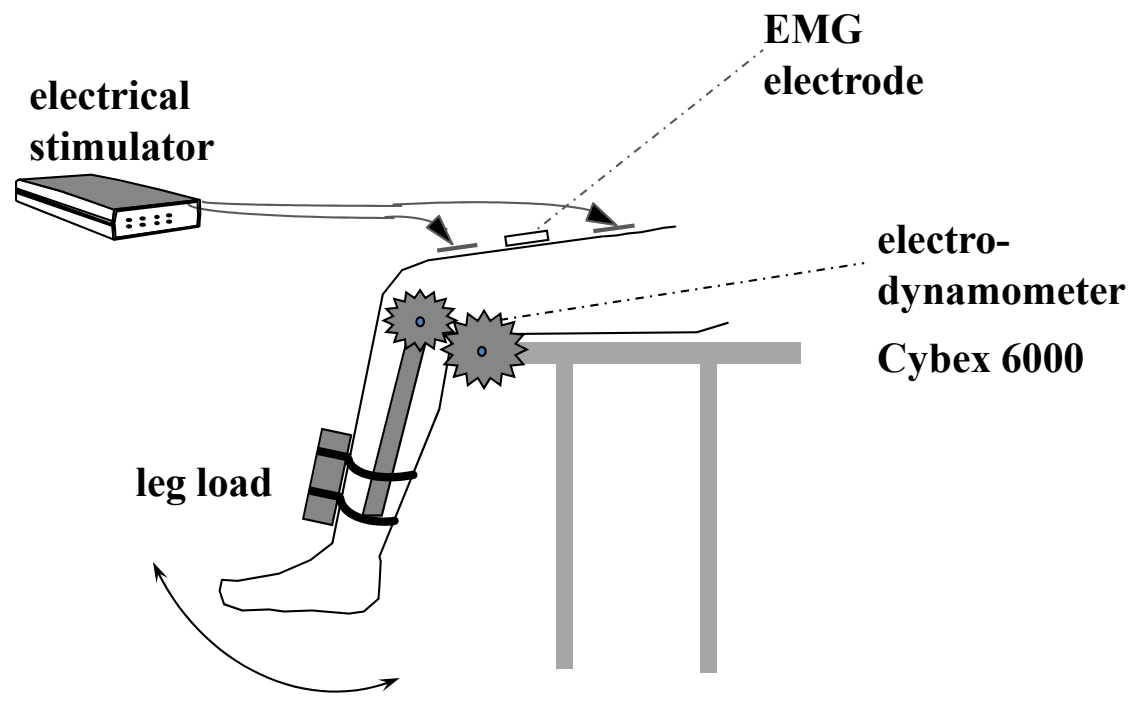

Fig. 2. Apparatuses for stimulation and data recording (knee extension torque and EMG).

of detecting and stimulating electrodes. Before the application of measurement electrodes, the skin was abraded carefully to reduce skin-electrode impedance. To suppress the stimulus artifact, the output stage of the stimulator was grounded immediately after the stimulus pulse was generated which was followed by artifact suppression algorithm [21]. The signal was differentially amplified with a gain of 380. After artifact suppression and amplification, the stimulus-evoked EMG was sampled through an analogue-to-digital converter with $5 \mathrm{kHz}$ sampling rate after an anti-aliasing filter at $2.5 \mathrm{kHz}$. The measured data were directly saved to a hard disk for later analysis using Matlab (Mathwork Co., Natick, Mass., USA) signal processing software.

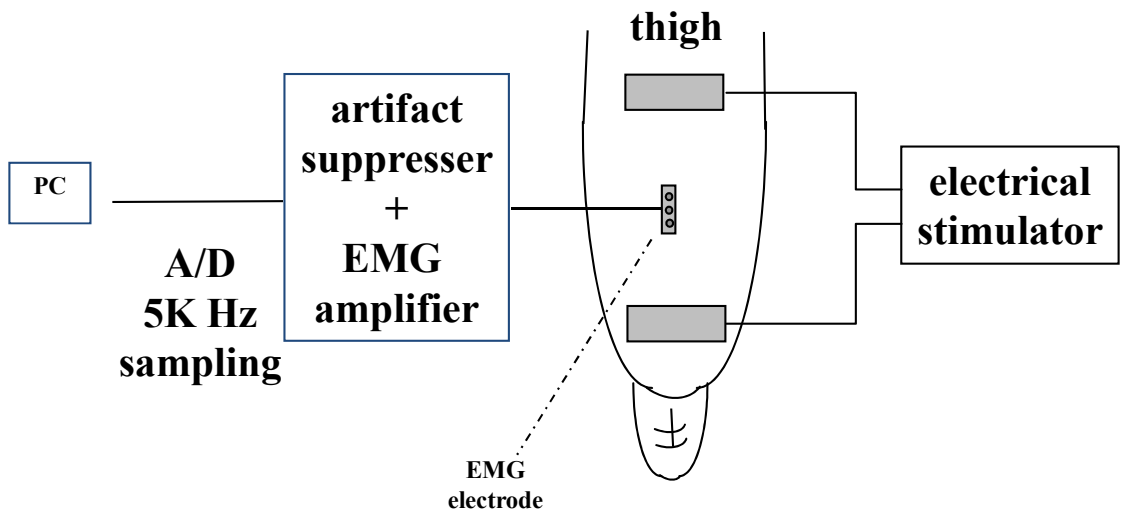

Fig. 3. The placement of EMG and stimulating electrodes. 


\subsection{Analysis of fatigue process from features of evoked EMG}

Both amplitude and temporal features are commonly used to characterize the waveform of the stimulus-evoked EMG. In a succession of sustained muscle contractions, the change in the amplitude parameter can be divided into the first plateau, a decaying slope, and the residual asymptotic level. Conversely, the changes of temporal parameters with inverted manifestation to the frequency parameter can be divided into an initial lower plateau, a rising slope, and the maximal asymptotic level. Our interest is in observing the rising and decaying processes of the CMAP parameters and the torque output during the fatigue process, rather than in making a direct comparison of their values. Thus, the data collected from the same session were first normalized to their initial value and then fitted using the hyperbolic function, which could also alleviate the inter-subject variations and musclelength discrepancies.

To characterize the fatigue process, a hyperbolic tangent equation, generally used for the description of empirical data with a sigmoid-like curve, was adopted to fit both the parameters of the CMAP and the torque output [8]:

$$
Y=a \times \tanh (b \times t-c)+d
$$

where $Y$ is the measured parameter of the CMAP or the generated torque as a function of time, $t$. The parameters $a, b, c$ and $d$ are estimated by using the least-square error method [8]. The inflection time and time constant, representing the time to reach $50 \%$ of the change range and its changing rate, can be obtained from $c / b$ and $1 / b$, respectively. Derived from the parameters, $d-a$ and $d+a$ represent the initial plateau and the asymptotic value respectively. The total change of the parameter (the difference between maximum and minimum values) is $2 a$. The value of $(d+a) /(d-a)$ represents the relative asymptotic value.

With the extracted parameters, muscle fatigue tests were performed in each lower limb at two different muscle lengths of all six SCI subjects, making a total of 24 experimental specimens of the stimulus EMG and torque. Since the normalization was performed, the effect of muscle length on the fatigue process was analyzed by comparing the time constant, inflection time and relative asymptotic value of the four measures including torque output, PTP, RTP, PTPd, between the two lengths $\left(30^{\circ}\right.$ and $110^{\circ}$ knee flexion). Paired $t$ tests were utilized to test the length effect. The significant level was defined as $\alpha<0.05$. Data are reported as means $\pm \mathrm{SD}$ within the text and the table.

\subsection{Analysis of fatigue process from muscle fiber components of evoked EMG}

Compared to the ordered recruitment of MUs in voluntary EMG, the CMAP is considered as the summation of the MUAPs simultaneous firing from different types of muscle fibers near the detecting electrode (Figure 4). Thus, the measured CMAP can be decomposed into the MUAPs with slow-twitch (type I) or fast-twitch (type II) characteristics. Figure 5 illustrates the propagation characteristics of MUAPs from type I or type II units. However, the technique to estimate the muscle fibers components from its summation, i.e. the CMAP, belongs to an inverse problem which requires a model to simulate the CMAP (called forward modeling) and an optimization technique to measure the goodness of resulting modeling parameters (called backward optimization). For the CMAP modeling, the generation of MUAP is adopted from structural EMG modeling of Fuglevand et al. [18] with some modifications to take in account of parameters for different muscle fiber types. Figure 6 depicts the schematic diagram of the CMAP signal generation mechanism which consists 
of slow-twitch and fast twitch fibers during electrically elicited contractions. To simplify the computational process, each motor unit territory area was crossed by a number of semicircular layers with an increment of $0.5 \mathrm{~mm}$ (equivalent to about 9 average fiber diameters), where each MU at the same layer is assumed to contribute the same potential to the detecting electrode.

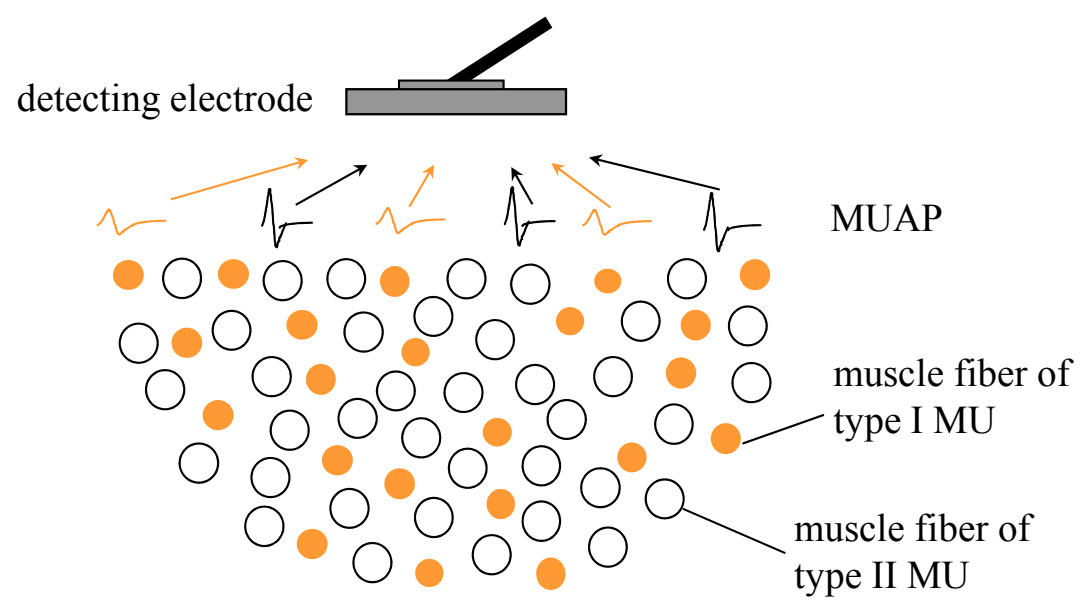

Fig. 4. The CMAP is composed of MUAPs simultaneously firing from the muscle fibers near the detecting electrode.

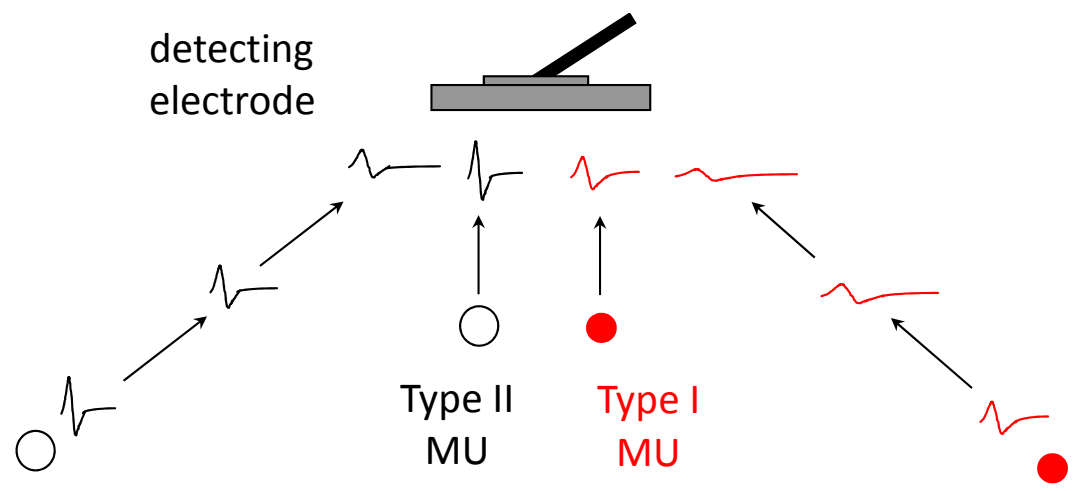

Fig. 5. Waveforms of MUAP at different distances from signal source. 


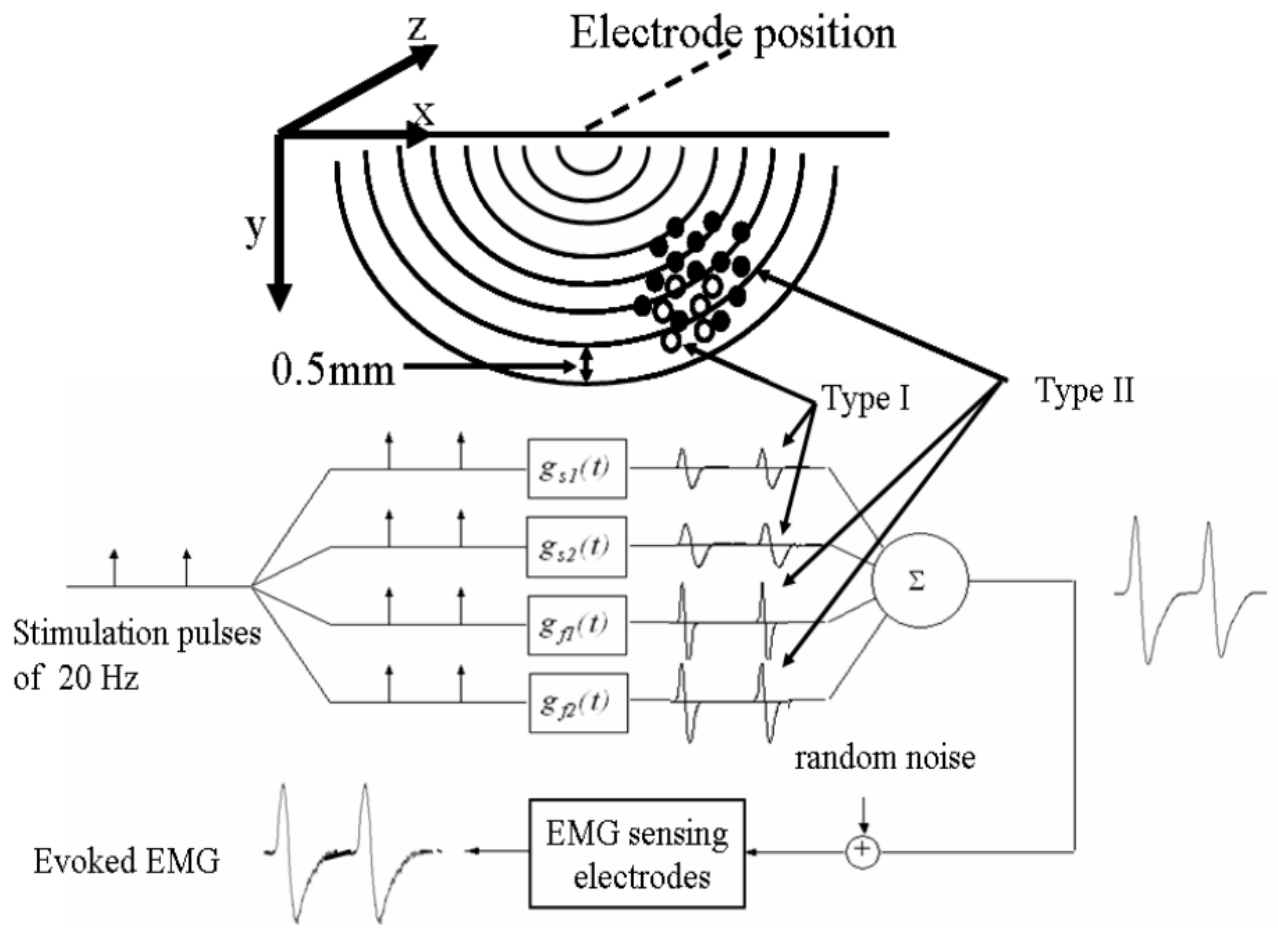

Fig. 6. Schematic diagram of the measured stimulus EMG can be modeled as the sum of MUAPs, with type I (slow-twitch) or type II (fast-twitch) characteristics, simultaneously firing from the muscle fibers randomly distributed within the detection range of surface EMG electrode. The generation of MUAP is based on structural EMG modeling developed by of Fuglevand et al. with some modifications to include two muscle fiber types of slowtwitch, $g_{s}(t)$ and fast twitch, $g_{f}(t)$. It is known that type I muscle fibers have smaller amplitude and slower conduction velocity with wide duration, compared with those of type II. In addition, type I muscle fibers (o) are located deeper and have lower distribution percentages (30\% type I vs. $70 \%$ type II) than those of type II muscle fibers $(\bullet)$ in chronic SCI subjects.

The function of a CMAP which comprises $m$ type I MUs and $n$ type II MUs can be formulated as:

$$
\mathrm{G}(\mathrm{t})=\sum_{\mathrm{i}=1}^{\mathrm{m}} k_{s} e^{-t_{s i} / \tau} g\left(b_{s i} \cdot\left(t-t_{s i}\right)\right)+\sum_{\mathrm{i}=1}^{\mathrm{n}} k_{f} e^{-t_{f i} / \tau} g\left(b_{f i} \cdot\left(t-t_{f i}\right)\right)
$$

where $g$ is an MUAP function with $\mathrm{m}$ fibers from slow-twitch MUs and $\mathrm{n}$ fibers from fasttwitch ones. Figure 7 shows the model of CMAP composed of type I and type II MUAPs. Parameters $k_{s}$ and $k_{f}$ are amplitude gains of type I and type II MUs respectively. The exponential term in equation 2 represents the amplitude gain of each MUAP which is inversely and exponentially proportional to the time delays $t_{s i}$ and $t_{f i}$ with a fixed decaying time constant $\tau$. The temporal coefficients $b_{s i}$ and $b_{f i}$ are used to determine the 
duration of $i$-th MUAP. The propagation time along the muscle fiber determines the time delay and the duration of an MUAP which are directly related to the conduction distance of the MUAP. Therefore, MUAPs from different distances have different amplitudes, durations and time delays which are all functions of the electrode-motor unit territory distance (ETD). When a conduction distance along the muscle fiber is longer, the amplitude of the evoked EMG decreases and the duration increases due to the desynchronization of single motor unit potential arrivals at the recording site [22]. Therefore, both amplitude and temporal coefficients are functions of the ETD, the simulation model can hence be formulated as:

$$
\mathrm{G}(d)=\sum_{\mathrm{r}=1}^{d} \mathrm{~A}(\mathrm{r}) e^{-r / \lambda}\left(\mathrm{k}_{s} g_{s}(r)+\mathrm{k}_{f} g_{f}(r)\right)
$$

where $g_{s}(r)$ and $g_{f}(r)$ represent the MUAPs of slow twitch (type I) and fast twitch (type II) muscle fibers from a distance of $r$, i.e. they are functions of ETD. The $r$ in equation 3 represents the radius of each semicircular layer, with the same origin at the center of the detecting electrode. Equation 3 comprises a number of functions which represent the MUAPs generated from their corresponding isopotential layers. The area of the $r$-th isopotential layer $A(r)$ is $\frac{1}{2} \pi(2 r-1)$.

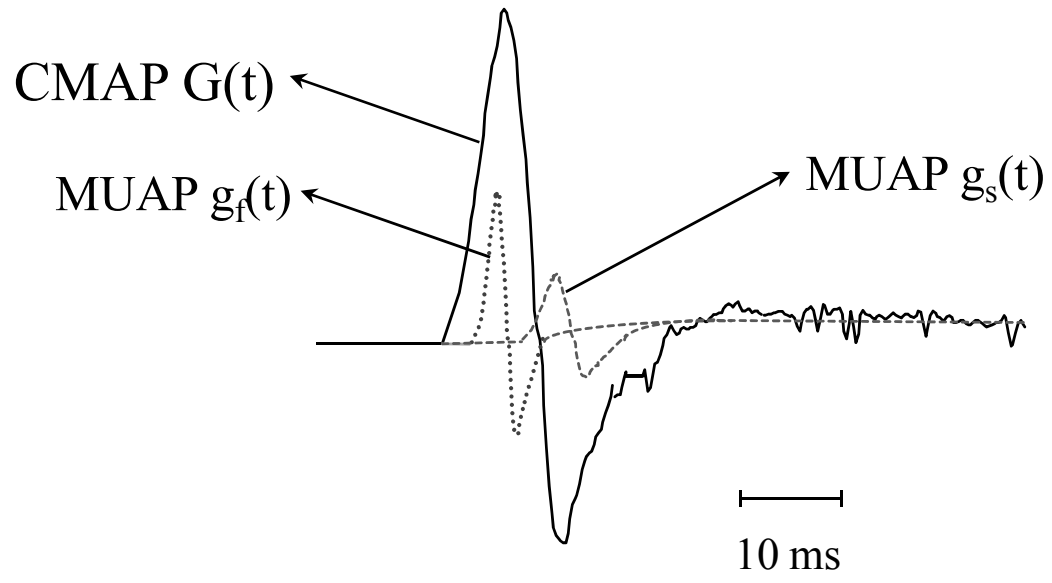

Fig. 7. Illustration of the Model of CMAP.

The generation of MUAP function $g$ can be obtained from the parameters provided by Fuglevand et al. [18] with some modifications to include slow-twitch and fast-twitch MUs. The ranges of the assigned parameters for the modeling of evoked-EMG from two types of muscle fibers are listed in Table 1. 
Muscle fiber types Type I (slow twitch) Type II (fast twitch)

Percentages of distribution

Amplitude

Initial value for gain coefficients before fatigue

Propagation velocity

Initial value for temporal coefficients before fatigue

\begin{tabular}{ll}
$30 \%$ & $70 \%$ \\
\hline Small & Large \\
$\mathrm{k}_{\mathrm{s}}=\left[\begin{array}{ll}0.03 & 0.09\end{array}\right]$ & $\mathrm{k}_{\mathrm{f}}=\left[\begin{array}{ll}0.05 & 0.15\end{array}\right]$ \\
\hline $1.5-3.0 \mathrm{~m} / \mathrm{s}$ & $4.5-9.0 \mathrm{~m} / \mathrm{s}$ \\
$b_{s}=\left[\begin{array}{lll}2.0 & 3.5\end{array}\right]$ & $b_{f}=\left[\begin{array}{ll}1.0 & 1.5\end{array}\right]$
\end{tabular}

Table 1. Parameters for the simulation of MUAPs for type I and type II muscle fibers.

First, researchers have shown that the distribution percentages of muscle fibers are around $30 \%$ (type I) versus $70 \%$ (type II) in chronic SCI cases, different from those of normal subjects with percentages of $52 \%$ (type I) versus $48 \%$ (type II) [23]. The proportion of MU type was fixed but the gain values, $\mathrm{k}_{s}$ and $\mathrm{k}_{f}$, were varied according to the general consensus that type I muscle fibers are smaller in amplitude and are located deeper than the type II muscle fibers. The ranges for $\mathrm{k}_{s}$ and $\mathrm{k}_{f}$ are listed in Table 1.

Second, the average conduction velocity for all fiber types, $4 \mathrm{~m} / \mathrm{s}$, was assigned in previous structural EMG model [18], which was divided into 1.5-3.0 m/s for type I and 4.5-9.0 m/s for type II muscle fibers [22]. In addition to elongated duration because of lower conduction velocity, the type I muscle fibers are usually located deeper which results in smaller amplitude in the surface recording. By identifying the relationship between the two pairs of temporal coefficients, the estimation process can be simplified to assess either $b_{s i}$ and $b_{f i}$ or $t_{s i}$ and $t_{f i}$. This is because the time delay $t_{s i}$ and $t_{f i}$ are related to the anisotropic conduction velocity of muscle fiber, whereas the coefficients $b_{s i}$ and $b_{f i}$, used for determining the duration of an MUAP, are directly related to the longitudinal conduction velocity. The radial conduction velocity of muscle fiber is about one sixth of the longitudinal conduction velocity, therefore the values of $t_{s i}$ and $t_{f i}$ can be assumed to depend on those of $b_{s i}$ and $b_{f i}$. The assigned values for $b_{s i}$ and $b_{f i}$ are inversely proportional to the physiological ranges of conduction velocity for Type I and Type II muscle fibers, as listed in Table 1. The estimation of the above parameters was obtained by fitting the measured data to the simulated function.

Among the structural EMG parameters, $k_{s}$ and $k_{f}$ are amplitude gain features of type I and type II muscle fibers respectively. Another set of important features are the temporal coefficients $b_{s i}$ and $b_{f i}$ which were used to determine the duration of every MUAP. The estimation of the above parameters was obtained by fitting the measured data to the simulated function. A nonlinear curve fitting technique was used to find the optimal parameters such that the error could be reduced to a minimum by using the Nelder-Mead simplex algorithm [24]. The parameters derived from the evoked-EMG during fatigue process of two different positions were compared. 


\section{Results}

\subsection{Characteristics of muscle fatigue at different knee angles}

Figures 8 shows the representative CMAP (dotted lines) measured from the shortened and lengthened muscles from the beginning of stimulation (10th second) and the fatigue situation (120th second), respectively. We can clearly observe that CMAPs before fatigue have a greater amplitude and more compact waveform compared to those with smaller amplitude and broader waveform measured from the 120th second. Comparing the CMAPs with different muscle lengths, the amplitude decreased and the duration of CMAP was elongated extensively in the lengthened muscles, as shown in Fig. 8(b).

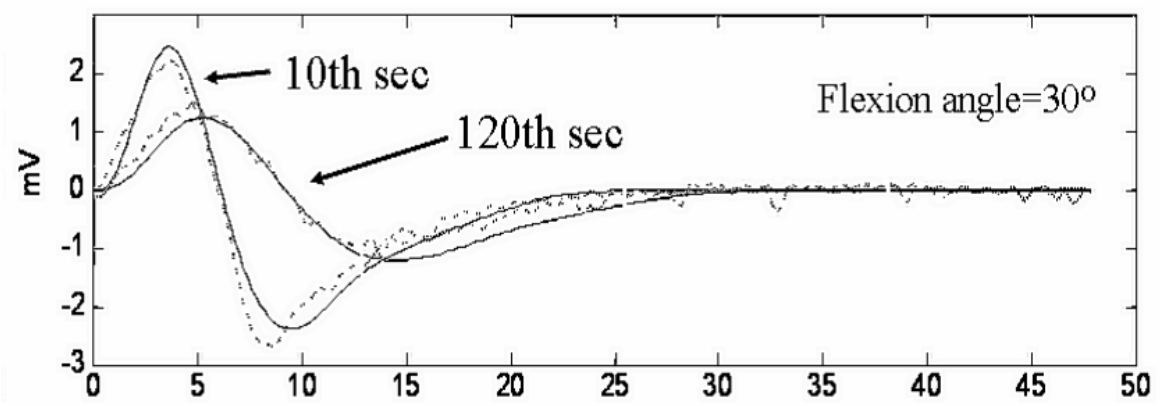

(a)

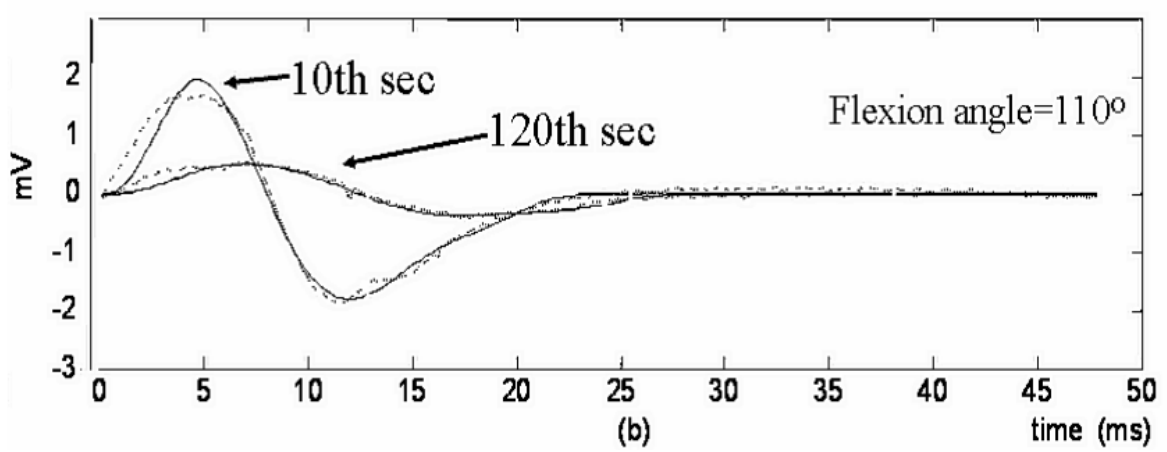

Fig. 8. Representative CMAP signals (dashed line) measured at the 10th and 120th seconds at (a) shortened and (b) lengthened positions. Compared to those measured in the 120th sec, CMAPs have higher amplitude and shorter duration before fatigue. In addition, CMAPs measured at shorter muscle length have short peak-to-peak duration in comparison with those of lengthened one. The solid lines represent the fitted curves which were generated from a structural EMG model consisting of two muscle fibers types.

The influence of muscle lengths on torque and EMG features can be observed from the measurements of isometric contractions measured at two different knee angles. The EMG amplitude (PTP) and torque output were normalized with respect to their maximum, but the EMG RTP and PTP duration were normalized with respect to their minimum. Figure 9 shows the means and standard deviations of the normalized torque output and the EMG 
features of the six SCI subjects for muscles stimulated at $30^{\circ}(-\mathrm{o}-)$ and $110^{\circ}(-\mathrm{x}-)$ of knee flexion. The corresponding parameters for fitting the sigmoid function to the curves are listed in Table 2.
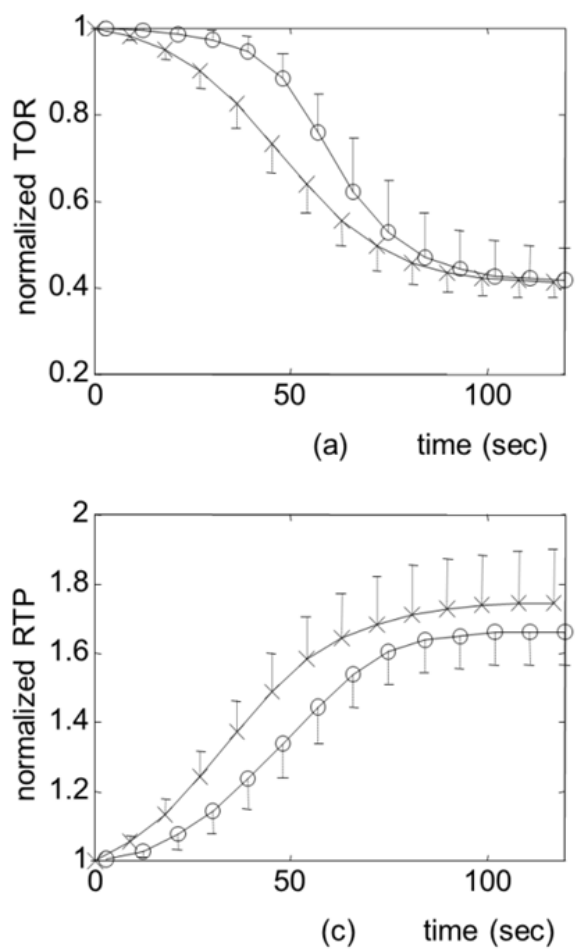
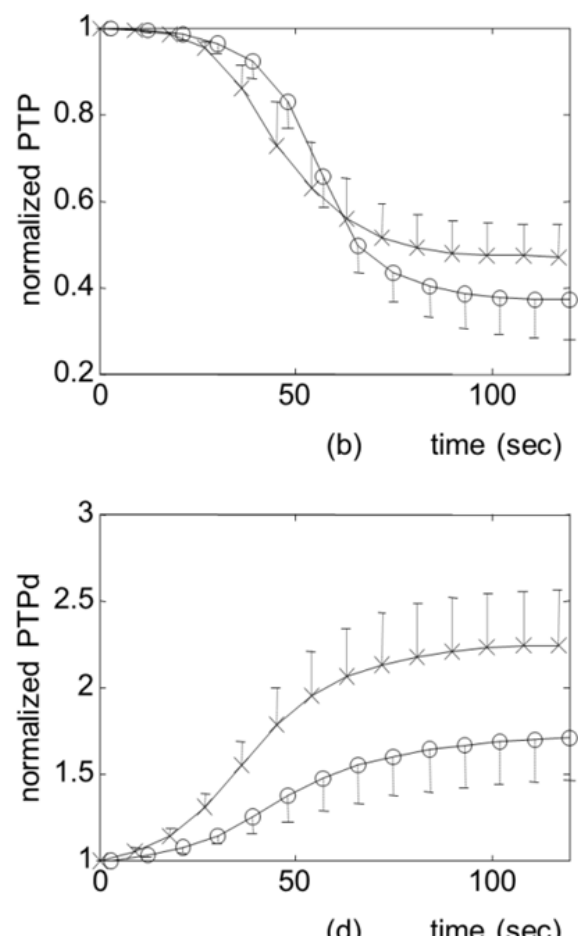

Fig. 9. The measurements of torque output (TOR) and EMG features after amplitude normalization are averaged for isometric contractions at knee flexion at $30^{\circ}(-\mathrm{o}-)$ and $110^{\circ}$ $(-x-)$, with standard deviations shown in vertical bars. The (a) torque output and (b) EMG PTP amplitude show decreasing trends while the (c) EMG RTP and (d) EMG PTP duration exhibit increasing trends during muscle fatigue. All the measurements are fitted to hyperbolic functions with parameters summarized in Table 2.

The torque measured in this process is used to assess when the stimulated-muscle reached the fatigue level, i.e. a plateau at a relatively lower torque output. Figure 9(a) shows that the torques in $30^{\circ}$ knee flexion (muscles in shortened length) has an initial plateau and then decreases at a fast rate to a lower plateau with a relative asymptotic value of 0.41 , derived from $\mathrm{d}+\mathrm{a} / \mathrm{d}-\mathrm{a}$. Torques from lengthened muscles $\left(110^{\circ} \mathrm{knee}\right.$ flexion) show no obvious initial plateau but decrease earlier at a slower rate to a similar asymptotic value of 0.39 . These observations can be confirmed from the significant differences in time constant $(20.79 \pm 4.43 \mathrm{~s}$ vs. $29.83 \pm 9.63 \mathrm{~s})$ and inflection time $(61.15 \pm 9.69 \mathrm{~s}$ vs. $49.12 \pm 8.83 \mathrm{~s})$ in torque output between the two flexion angles (Table 2).

Similarly, in EMG PTP amplitude, the significantly smaller inflection time of a $110^{\circ}$ flexion angle $\left(48.53 \pm 8.70 \mathrm{~s}\right.$ compared to $55.13 \pm 4.03 \mathrm{~s}$ at $\left.30^{\circ}\right)$ indicates that the lengthened muscle fatigues earlier than the shortened muscle. A relatively close time constant and asymptotic 


\begin{tabular}{llll}
\hline & $\begin{array}{l}\text { Time constant } \\
(1 / \mathrm{b})(\mathrm{sec})\end{array}$ & $\begin{array}{l}\text { Inflection time } \\
(\mathrm{c} / \mathrm{b})(\mathrm{sec})\end{array}$ & $\begin{array}{l}\text { Relative asymptotic value } \\
(\mathrm{d}+\mathrm{a}) /(\mathrm{d}-\mathrm{a})\end{array}$ \\
\hline $\mathrm{TOR}_{30}$ & $20.79(4.43)^{*}$ & $61.15(9.69)^{*}$ & $0.41(0.10)$ \\
$\mathrm{TOR}_{110}$ & $29.83(9.63)^{*}$ & $49.12(8.83)^{*}$ & $0.39(0.05)$ \\
$\mathrm{PTP}_{30}$ & $15.87(9.54)$ & $55.13(4.03)^{*}$ & $0.39(0.14)$ \\
$\mathrm{PTP}_{110}$ & $15.56(4.87)$ & $48.53(8.70)^{*}$ & $0.45(0.13)$ \\
$\mathrm{RTP}_{30}$ & $35.33(9.92)$ & $48.44(11.93)^{*}$ & $1.64(0.31)$ \\
$\mathrm{RTP}_{110}$ & $30.39(7.48)$ & $35.49(6.41)^{*}$ & $1.75(0.63)$ \\
$\mathrm{PTPd}_{30}$ & $32.03(9.57)^{*}$ & $46.39(9.55)^{*}$ & $1.77(0.54)^{*}$ \\
$\operatorname{PTPd}_{110}$ & $24.71(8.12)^{*}$ & $36.40(12.63)^{*}$ & $2.23(0.74)^{*}$ \\
\hline
\end{tabular}

$\mathrm{PTP}=$ peak to peak amplitude, RTP = rise time to peak, PTPd = peak to peak duration. The subscript ' 30 ' and ' 110 ' denote the knee flexion angles. The '*' denotes the difference $(\alpha<0.05)$ between the parameters measured at two knee flexion angles.

Table 2. Summary of hyperbolic fit parameters for the fatigue process during isometric contractions at two knee flexion angles $\left(30^{\circ}\right.$ and $\left.110^{\circ}\right)$. The values in brackets are the corresponding standard deviations.

value can be observed in the PTP amplitudes of the two flexion angles (Table 2 and Fig. 9(b)). Our results also show that both the RTP and PTPd of the lengthened muscles increase earlier (with a shorter inflection time of $35.49 \pm 6.41$ and $36.40 \pm 12.63$, respectively) than those of shortened muscles (48.44 \pm 11.93 and $46.39 \pm 9.55$, respectively), as depicted in Fig. 9(c) and 9(d). The relative asymptotic value of PTPd in lengthened RF muscle is higher than that in the shortened position (2.23 \pm 0.74 vs. $1.77 \pm 0.54)$, as shown in Fig. 9(d).

\subsection{Analysis of fatigue process based on muscle fiber components}

To estimate the amplitude and temporal coefficients form the CMAP, the initial values of amplitude gains $\left(k_{s}\right.$ and $\left.k_{f}\right)$ and temporal coefficients $\left(b_{s}\right.$ and $\left.b_{f}\right)$ were initially assigned according to the physiological values, as listed in Table I. In general, the slow twitch muscle fibers have smaller amplitude (smaller $k_{s}$ ) and lower conduction velocity (greater $b_{s}$ ), compared to those of fast twitch muscle fibers [22]. After the assignment of initial values, the nonlinear curve fitting technique was used to estimate those values from each CMAP measured during fatigue process. As shown in Fig. 8, the estimated MUAP waveforms (solid lines) closely resemble to the measured ones (dotted lines) with an average relative error smaller than $5 \%$ of the measured MUAP.

Feature extraction of muscle fiber components was performed with the curve fitting technique on the measured CMAP for duration of $120 \mathrm{~s}$. The averages of amplitude coefficients of slow-twitch $\left(k_{s}\right)$ and fast-twitch $\left(k_{f}\right)$ for shortened and lengthened muscle positions are shown in Fig. 10, where the dashed and solid curves show the changes of $k_{s}$ and $k_{f}$ respectively. At the beginning of stimulation, amplitude coefficients of fast twitch are greater than those of slow twitch, which is even evident in RF muscle in a shortened 
condition (Fig. 10(a)). With the time elapse of stimulation, both of the coefficients in the shortened muscle increased first then decreased into a residual value (Fig. 10(a)). In the lengthened muscle, both the coefficients decreased near monotonically to a lower residue value as shown in Fig. 10(b). When comparing their decreasing speeds, the amplitude coefficient in type II MU dropped abruptly into a lower plateau at around 40 seconds after the beginning of stimulation (Fig. 10(b)). Although the declining curve was not so steep in the shortened muscles, the apparent drops of the amplitude coefficients in the type II MU can also be noted. In addition, the amplitude gains of type I MU dropped to $33 \%$ and $30 \%$ for shortened and lengthened muscles. In contrast, those of type II MU dropped to $15 \%$ and $13 \%$ of their original values. The amplitude gain coefficients of slow twitch became larger than those of fast twitch, at the last period of stimulation around $80^{\text {th }} \mathrm{s}$ in shortened position and after $40^{\text {th }} \mathrm{s}$ in lengthened position.

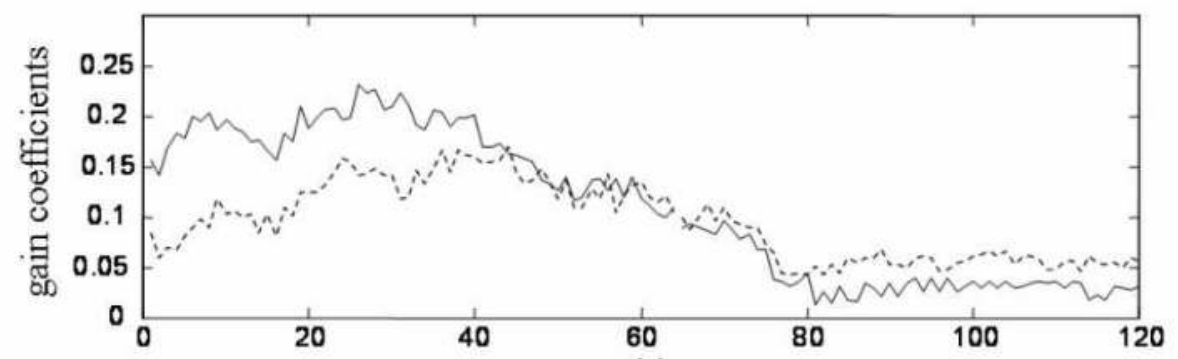

(a)

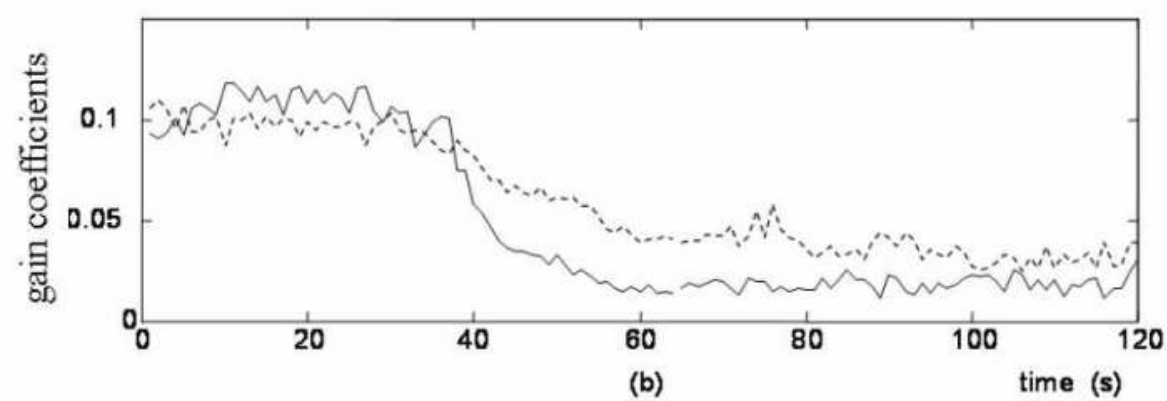

Fig. 10. Changes in amplitude gain parameters from the nonlinear curve fitting of CMAP measured at (a) shortened and (b) lengthened muscles for slow twitch (dashed line) and fast twitch, (solid line) muscle fibers, respectively.

The changes of average temporal coefficients for type I $\left(b_{s}\right)$ (dashed line) and type II $\left(b_{f}\right)$ (solid line) for two muscle lengths are shown in Fig. 11. It is evident that temporal coefficients $b_{s}$ of type I muscle fibers are larger than those of type II in both shortened and lengthened positions. In shortened muscles, the $b_{s}$ and $b_{f}$ coefficients started at around 1.9 and 1.1 which increased monotonically into an upper plateau around 2.9 and 1.8, respectively, Similar observations can be found in lengthened muscles which increased from 2.2 to 5.0 for $b_{s}$ and from 1.5 to 3.8 for $b_{f}$, respectively. In addition, the increasing rate of $b_{s}$ and $b_{f}$ is higher for lengthened muscles, compared to that of shortened one. However, the 
increasing speed of two temporal coefficients at a same flexion angle does not show significant difference.

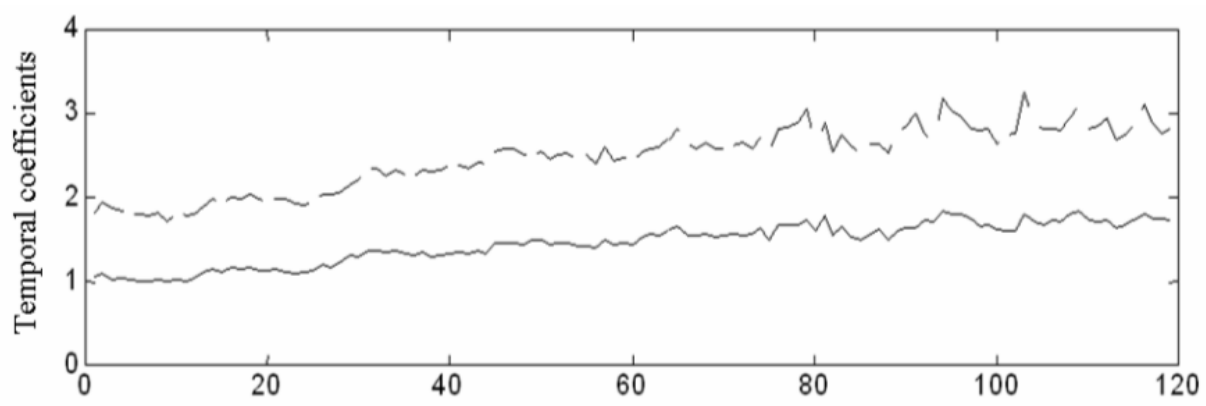

(a)

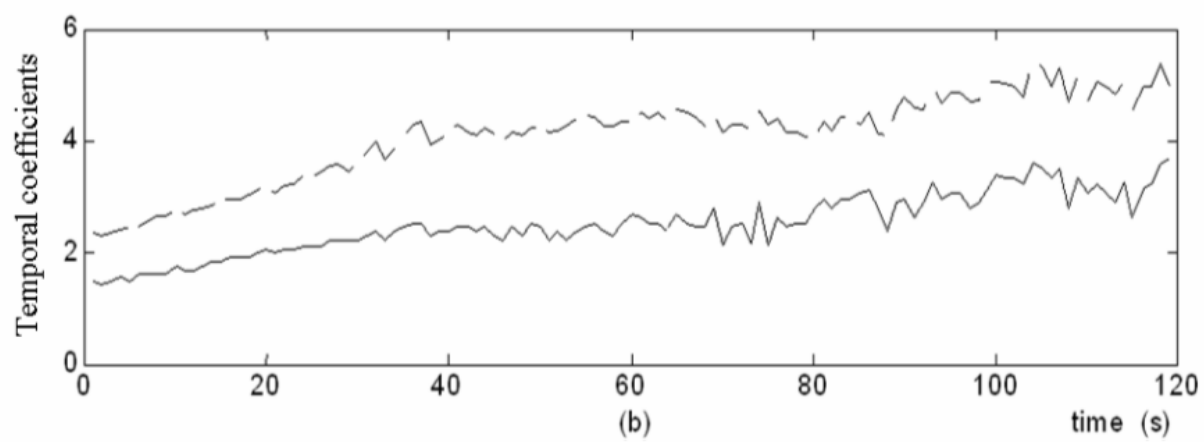

Fig. 11. The extraction of temporal coefficients from the measured CMAP of (a) shortened and (b) lengthened muscles during 120 seconds of fatigue process. Increasing trend in the temporal coefficients for slow twitch $\left(b_{s}\right.$, dashed line) and fast twitch $\left(b_{f}\right.$, solid line) muscle fibers can be observed.

\section{Discussion}

\subsection{Effect of muscle length on the fatigue process}

Under continuous stimulation, the EMG PTP amplitude exhibited a decreasing trend which exhibited a sigmoid-like curve with a main slope and two plateaus during muscle fatigue. Concurrently with the reduction in EMG PTP amplitude, the increase in RTP and PTP duration denotes a broadening of the CMAP, which is commonly explained by the reduction of the muscle fiber's conduction velocity during the fatigue process [7].

Among the amplitude and temporal parameters, our results showed a larger inflection time for the four measured features in shortened muscles, compared to those in lengthened muscles (Table 2). Previous studies on the effect of muscle length on temporal features revealed differences in conduction velocity estimates in muscles with different lengths [13, 17]. Differences in amplitude features were also found at different muscle lengths [16]. When a muscle is shortened, assuming no or little change in the volume of muscle fibers, the conduction velocity must be increased because of the larger diameter of muscle fibers with 
lower conducting impedance [13]. Action potentials propagating along muscle fibers with faster conduction velocities are detected more nearly simultaneously, causing the CMAP to appear larger and more compressed. During the fatigue process, the conduction velocity in T-tube and sarcoplasmic reticulum is reduced such that the propagation time in the muscle fiber is further prolonged [25]. The longer the muscle fiber, the more difficult the propagation in the muscle fibers, resulting in earlier and faster fatigue. Researchers have also indicated that the fatigue-resistant characteristics can be found in shortened muscles of healthy subjects [26]. These phenomena were interpreted that a reduced force in shortened muscles reflects a reduced number of cross-bridge interactions and a lower total ATP turnover. Other researchers studied the effects of length on the change of contractile properties [27] and stimulation frequency on the fatigue properties [25]. They found similar fatigue properties in shortened muscles and high frequency stimulation, and attributed the phenomena to the failure of sarcolemmal action potential propagation in the transverse tubules of shortened muscles.

\subsection{Physiological implications of muscle fiber components during muscle fatigue}

It is known that different types of MU have their own features in the electrically-elicited myoelectric signal [28, 29]. The action potential propagation velocity in muscle fiber is known to be related to the type and diameter of muscle fiber [29], to the extracellular potassium concentration, and to the $\mathrm{pH}$ of intracellular or interstitial fluid [30]. Research has shown that fast-twitch MUs display a marked reduction in amplitude and the integrated area, whereas the slow-twitch MUs shows little or no change in surface action potential during muscle fatigue [28]. Other studies indicated that the decreasing curves of the EMG amplitude could be the reflection of the activation of the residual fatigue-resistant MUs, which have small amplitude in MUAPs [7]. Thus, the distinct components in the rising sigmoid curve of the temporal features of CMAP might not only denote the reduction in the conduction velocity but also indicate which type of MU is predominating during the muscle fatigue process. Our analysis of fatigue process from the muscle fiber components supported this observation.

The amplitude gains in the shortened muscle increased first then decreased monotonically. It seems that the electrical activity of both types of MUs were initially potentiated and then released gradually in the shortened muscle. This phenomenon could be ascribed to a process of synchronized MUAPs which matches the early plateau observed in the aspects of both torque output and the EMG PTP (Fig. 9(a) and (b)). Contrary to the shortened muscles, the lengthened muscles exhibited a slow decrease in amplitude gain at a prolonged duration during the first 40 second of stimulation. After that, the amplitude gain coefficients of type II MU dropped abruptly into a lower plateau, especially in when the muscles were stimulated in the lengthened positions. These results implied that early fatigue in lengthened muscles might be due to the quick depletion of the type II MUs. Through making a comparison between the two types of MU, the more intense decline of the amplitude coefficient of type II MU especially in lengthened muscle (Fig. 10(b)) indicates its contribution to the early muscle fatigue. From the slower declining speed of the amplitude coefficient of type I MU, the greater amplitude gain implying more dominant contribution from the fatigue-resistant muscle fibers could be noted in the late period of stimulation (Fig. 10).

The temporal coefficients $b_{s}$ and $b_{f}$ of two muscle lengths show steady increase during the fatigue process, differently from the amplitude coefficients. The increasing rate of $b_{s}$ and $b_{f}$ 
is higher for lengthened muscles (Fig. 11(b)), compared to that of shortened one (Fig. 11(a)). An increase in temporal coefficient means the elongated duration of the CMAP and the decrease in conduction velocity during muscle fatigue in both muscle types. The changes of temporal coefficients in type I and type II MUs (Fig. 11) of two muscle lengths exhibited similar characteristics when compared with the temporal PTPd features of the CMAP (Fig. 9 (d)), which showed a higher increasing rate with lower time constant and higher relative asymptotic value for lengthened muscle (Table 2).

Our results supported that the sigmoid-like curves in the CMAP features might correlate with the changed contributions of two MU types. The smaller RTP and PTP duration in the first plateau (Fig. 9(c) and (d)) and larger amplitude coefficients (Fig. 10) in fast twitch muscle fibers indicate that type II MUs with larger diameter muscle/nerve fibers and fast conduction velocity are those predominantly activated in early electrical stimulation period. By contrast, type I MUs with lower conduction velocities are more resistant to fatigue and might, therefore, be predominant in the EMG signal when muscle fatigue progresses [31]. This predominance tends to decrease the average muscle fiber conduction velocity, and then to elevate the curves of the temporal features to the final plateau. These phenomena can also be observed from larger amplitude coefficient and higher residual values for type I MUs (Fig. 10) during muscle fatigue at the end of stimulation period.

\section{Conclusions}

The hyperbolic curve fit of CMAP features as well as the extracted coefficients of muscle fiber components were used to observe muscle fatigue induced by continuous electrical stimulation at two different muscle lengths. Our results showed that RF muscle is more easily fatigable when lengthened than when shortened, which can be observed from shorter inflection times of CMAP features and from abrupt decreasing trend in gain coefficient of type II MUs. Compared to type II MUs, the amplitude gain coefficients of type I MUs started at smaller values but slowly dropped to a plateau level which was higher than those of fast twitch ones. These observations correlate well with the predominance of fast twitch type II MUs at the beginning of stimulation but the fatigue resistant type I MUs predominate the fatigue process at the end of stimulation period. . The higher increasing rate and larger final values of temporal coefficients in lengthened muscles, compared to that of shortened one, exhibited similar characteristics to the temporal PTPd features of the CMAP during fatigue process. However, our estimation of muscle fiber components was based a structural EMG model of Fuglevand et al. by adding additional parameters to specify the numbers, amplitude, and temporal coefficients for two different types of MUs. With more information acquired from the configuration and proportion of the MU types in paralyzed limb, constructing a sophisticated model of CMAP consisting for varied distributions of muscle types will be a challenge for future works. The interpretation from the distribution of muscle fiber components during fatigue process might become feasible by integrating the experimental design and evoked-EMG modeling.

\section{References}

[1] J. Mizrahi, "Fatigue in muscles activated by functional electrical stimulation," Crit. Rev. Phys. Rehabil. Med., vol. 9, pp. 93-129, 1997. 
[2] Y. Giat, J. Mizrahi, and M. Levy, "A model of fatigue and recovery in paraplegic's quadriceps muscle subjected to intermittent FES," J. Biomech. Eng., vol. 118, pp. 357-366, 1996.

[3] M. Levy, T. Kushnir, J. Mizrahi, and Y. Itzchak, “In vivo ${ }^{31 P}$ NMR studies of paraplegics' muscles activated by functional electrical stimulation. Magn. Res. Med., vol. 29, pp.53-58, 1993.

[4] J. Mizrahi, M. Levy, H. Ring, E. Isakov, and A. Liberson, "EMG as an indicator of fatigue in isometrically FES-activated paralyzed muscles," IEEE Trans. Rehab. Eng., vol. 2, pp. 57-65, 1994.

[5] B. K. Boom, A. J. Mulder, and P. H. Veltink, "Fatigue during functional neuromuscular stimulation," Progr. Brain Res., vol. 97, pp.409-418, 1993.

[6] E. Rabischong and F. Ohanna, "Effects of functional electrical stimulation on evoked muscular output in paraplegic quadriceps muscle," Paraplegia, vol. 30, pp. 467-473, 1992.

[7] E. Rabischong, "Surface action potential related to torque output in paraplegics electrically stimulated quadriceps muscle," Med. Eng. Phys., vol. 18, pp.538-547, 1996.

[8] E. Rabischong and D. Guiraud, "Determination of fatigue in the electrically stimulated quadriceps muscle and relative effect of ischemia," J. Biomed. Eng., vol. 15, pp.443450, 1993.

[9] W. Girsch, M. Bijak, G. Heger, R. Koller, H. Lanmuller, W. Mayr, H. Thomas, and U. Losert, "Monitoring of FES-induced muscle-activity by continuous EMGrecording," Int. J. Artif. Org., vol. 18, pp.340-244, 1995.

[10] J. Winslow, P. L. Jacobs, and D. Tepavac, "Fatigue compensation during FES using surface EMG," J. Electromyogr. Kinesiol., vol. 13, no. 6, pp.555-568, 2003.

[11] M. Bilodeau, J. Houck, T. Cuddeford, S. Sharma, and N. Riley, "Variations in the relationship between the frequency content of EMG signals and the rate of torque development in voluntary and elicited contractions," J. Electromyogr. Kinesiol., vol. 12, no. 2, pp. 137-145, 2002.

[12] N. C. Chesler and W. K. Durfee, "Surface EMG as a fatigue indicator during FESinduced isometric muscle contractions," J. Electromyogr. Kinesio., vol. 7, pp. 27-37, 1997.

[13] R. Merletti, L. R. Lo Conte, C. Cisari, and U. Massazza, "Effect of ankle joint position on electrically evoked surface myoelectric signals of the tibialis anterior muscle," Arch. Phys. Med. Rehabil., vol. 74, pp. 501-506, 1993.

[14] D. Farina, M. Fosci, and R. Merletti, "Motor unit recruitment strategies investigated by surface EMG variables: An experimental and model based feasibility study," J. Appl. Physiol., vol. 92, pp. 235-247, 2002.

[15] W. Muhammad, O. Meste, H. Rix, and D. Farina, "A pseudo joint estimation of time delay and scale factor for M-wave analysis," IEEE Trans. Biomed. Eng. Vol. 50, no. 4, pp. 459-468, 2003.

[16] J. J. Chen and Y. N. Yu, “The validity of stimulus-evoked EMG for studying muscle fatigue characteristics of paraplegic subjects during dynamic cycling movement," IEEE Trans. Rehab. Eng., vol. 5, pp. 170-178, 1997.

[17] D. Farina, R. Merletti, M. Nazzaro, and I.Caruso, "Effect of joint angle on EMG variables in leg and thigh muscles: what influence does muscle length and 
electrode location have on EMG signals during dynamic tasks?," IEEE Eng. Med. and Bio., vol. 20, pp. 62-71, 2001.

[18] J. Fuglevand, D. A.Winter, A. E. Patla, and D. Stashuk, "Detection of motor unit action potentials with surface electrode size and spacing," Biol. Cybern., vol. 67, pp. 14353, 1992.

[19] D. Farina and R. Merletti, "A novel approach for precise simulation of the EMG signal detected by surface electrode," IEEE Trans. Biomed. Eng., vol. 48, pp.637-646, 2001.

[20] ] E. F. Delagi and A. Perotto, Anatomic guide for the electromyographer: the limbs. 2nd ed., Thomas, Springfield, 1980.

[21] J. Minzly, J. Mizrahi, N. Hakim, and A. Liberson, “A stimulus artifact suppresser for EMG recording during FES by a constant-current stimulator," Med. Biol. Eng. Comput., vol. 31, pp.71-75, 1993.

[22] Y. Okajima, N. Chino, A. Tsubahara, and A. Kimura, "Waveform analysis of compound nerve action potentials: A computer simulation," Arch. Phys. Med. Rehabil., vol. 75, pp.960-964, 1994.

[23] ] R. Scelsi, "Skeletal muscle pathology after spinal cord injury: our 20 year experience and results on skeletal muscle changes in paraplegics, related to functional rehabilitation," Basic Appl. Myol., vol. 11, no.2, pp.75-85, 2001.

[24] J. C. Lagarias, J. A. Reeds, M. H. Wright, and P. E. Wright, "Convergence properties of the Nelder-Mead simplex method in low dimensions," SIAM Journal on Optimization, vol. 9, no. 1, pp. 112-147, 1998.

[25] P. Sacco, D. B. McIntyre, and D. A. Jones, "Effects of length and stimulation frequency on fatigue of the human tibialis anterior muscle," J. Appl. Physiol., vol. 77, pp.1148$1154,1994$.

[26] R. D. Kooistra, C. J. de Ruiter, and A. de Haan, "Muscle activation and blood flow do not explain the muscle length-dependent variation in quadriceps isometric endurance," vol. 97, pp. 1693-1701, J. Appl. Physiol., vol. 98, pp.810-816, 2005.

[27] ] N. Place, N. A. Maffiuletti, Y. Ballay, and R. Lepers, "Twitch potentiation is greater after a fatiguing submaximal isometric contraction performed at short vs. long quadriceps muscle length," J. Appl. Physiol., vol. 98, pp.429-436, 2005.

[28] K. Hainaut and J. Duchateau, "Muscle fatigue, effects of training and disuse," Muscle Nerve, vol. 12, pp.660-669, 1989.

[29] M. Knaflitz, R. Merletti, and C. J. DeLuca, "Inference of motor unit recruitment order in voluntary and electrically elicited contractions," J. Appl. Physiol., vol. 68, pp.1657$1667,1990$.

[30] C. Juel, "Muscle action potential propagation velocity changes during activity," Muscle Nerve, vol. 11, pp. 714-719, 1988.

[31] L. Arendt-Nielsen, K. R. Mills, and A. Forster, “Changes in muscle fiber conduction velocity, mean power frequency, and mean EMG voltage during prolonged submaximal contractions," Muscle Nerve, vol. 12, pp. 493-497, 1989. 


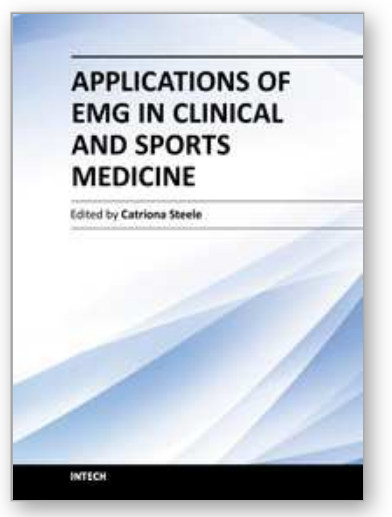

\author{
Applications of EMG in Clinical and Sports Medicine \\ Edited by Dr. Catriona Steele
}

ISBN 978-953-307-798-7

Hard cover, 396 pages

Publisher InTech

Published online 11, January, 2012

Published in print edition January, 2012

This second of two volumes on EMG (Electromyography) covers a wide range of clinical applications, as a complement to the methods discussed in volume 1. Topics range from gait and vibration analysis, through posture and falls prevention, to biofeedback in the treatment of neurologic swallowing impairment. The volume includes sections on back care, sports and performance medicine, gynecology/urology and orofacial function. Authors describe the procedures for their experimental studies with detailed and clear illustrations and references to the literature. The limitations of SEMG measures and methods for careful analysis are discussed. This broad compilation of articles discussing the use of EMG in both clinical and research applications demonstrates the utility of the method as a tool in a wide variety of disciplines and clinical fields.

\title{
How to reference
}

In order to correctly reference this scholarly work, feel free to copy and paste the following:

Shao-Hsia Chang and Nan-Ying Yu (2012). Experimentation and Structural Modeling of Stimulus-Evoked Electromyography in Muscles During Electrically-Elicited Fatigue Process, Applications of EMG in Clinical and Sports Medicine, Dr. Catriona Steele (Ed.), ISBN: 978-953-307-798-7, InTech, Available from: http://www.intechopen.com/books/applications-of-emg-in-clinical-and-sports-medicine/experimentation-andstructural-modeling-of-stimulus-evoked-electromyography-in-muscles-during-electr

\section{INTECH}

open science | open minds

\author{
InTech Europe \\ University Campus STeP Ri \\ Slavka Krautzeka 83/A \\ 51000 Rijeka, Croatia \\ Phone: +385 (51) 770447 \\ Fax: +385 (51) 686166 \\ www.intechopen.com
}

\author{
InTech China \\ Unit 405, Office Block, Hotel Equatorial Shanghai \\ No.65, Yan An Road (West), Shanghai, 200040, China \\ 中国上海市延安西路65号上海国际贵都大饭店办公楼 405 单元 \\ Phone: +86-21-62489820 \\ Fax: +86-21-62489821
}


(C) 2012 The Author(s). Licensee IntechOpen. This is an open access article distributed under the terms of the Creative Commons Attribution 3.0 License, which permits unrestricted use, distribution, and reproduction in any medium, provided the original work is properly cited. 